\title{
Purification and Characterization of a Major Human Pneumocystis carinii Surface Antigen
}

\author{
Bettina Lundgren, Gregg Y. Lipschik, and Joseph A. Kovacs \\ Critical Care Medicine Department, Clinical Center, National Institutes of Health, Bethesda, Maryland 20892
}

\begin{abstract}
Previous studies of Pneumocystis carinii have identified the major surface antigen of rat and human isolates as proteins of 116,000 and $95,000 \mathrm{~mol} \mathrm{wt}$, respectively, that are antigenically not identical. In this study both rat and human $P$. carinii proteins were purified by solubilization with zymolyase followed by molecular sieve and ion exchange chromatography. The native proteins had an apparent mol wt of 290,000 or greater, based on molecular sieve studies as well as cross-linking studies. Both proteins were glycoproteins; treatment with endoglycosidase $\mathrm{H}$ resulted in a $9 \%$ decrease in mol wt. The carbohydrate composition of the rat $P$. carinii glycoprotein was distinct from the human isolate; glucose, mannose, galactose, and glucosamine occurred in approximately equimolar ratios in the human $P$. carinii protein, whereas glucose and mannose were the predominant sugars of the rat $P$. carinii protein. To evaluate humoral immune responses to the human $P$. carinii protein, an enzyme-linked immunosorbent assay using purified protein was developed. Some, but not all, patients who subsequently developed $P$. carinii pneumonia demonstrated a serum antibody response to the surface antigen. Nearly all subjects without a history of $\boldsymbol{P}$. carinii pneumonia had no detectable antibodies. Purified $P$. carinii proteins will greatly facilitate the investigation of host-P. carinii interactions. (J. Clin. Invest. 1991. 87:163-170.) Key words: AIDS • immunosuppression • Pneumocystis carinii pneumonia • gp95 • ELISA
\end{abstract}

\section{Introduction}

Pneumocystis carinii pneumonia is a major, life-threatening complication of immunodeficiency diseases, especially AIDS (1). Although the incidence of this disease has increased dramatically in the past decade, $P$. carinii-host interactions remain poorly understood, and investigations into such interactions have primarily used organisms obtained from a rat model of $P$. carinii pneumonia. Antigens specific for $P$. carinii have recently been identified by the immunoblot technique, and antigenic differences between rat and human isolates have been demonstrated using monoclonal and polyclonal antibodies (26). Use of $P$. carinii-specific antigens in further studies has been limited by difficulties in purifying these antigens.

Studies of rat and ferret $P$. carinii have identified the major surface antigen as a glycoprotein with a molecular weight as

\footnotetext{
Address reprint requests to Dr. Joseph Kovacs, Critical Care Medicine Department, National Institutes of Health, Building 10, Room 10D48, Bethesda, MD 20892. 1990.

Received for publication 1 June 1990 and in revised form 22 August
}

The Journal of Clinical Investigation, Inc.

Volume 87, January 1991, 163-170 determined by SDS-PAGE of 110,000-120,000; this protein is frequently referred to as $\mathrm{gp} 116^{1}(7-11)$. There appears to be an analogous protein in human $P$. carinii with a mol wt of $\sim 95,000-98,000$ that is the major protein based on Coomassie-blue staining of SDS-PAGE gels $(2,4)$. Our laboratory has developed a monoclonal antibody (6B8) to this protein, and immunofluorescence studies have documented that this is a surface antigen (3). Moreover, this protein is different from the analogous rat $P$. carinii protein since 6B8 does not react with the rat $P$. carinii antigen (3). Since studies investigating the interaction of $P$. carinii with humans should use antigens derived from human $P$. carinii, we undertook to purify and characterize this human $P$. carinii surface antigen, and to develop an ELISA to measure antibody responses to this antigen. To obtain further information about the relationship between rat and human $P$. carinii, we simultaneously characterized the rat $P$. carinii antigen.

\section{Methods}

Organisms. Rat $P$. carinii were obtained from lungs of Sprague-Dawley rats treated with dexamethasone for 6 to 12 wk (12). Organisms were partially purified by Ficoll-Paque (Pharmacia Fine Chemicals, Piscataway, $\mathrm{NJ}$ ) density gradient centrifugation as previously described, washed in PBS pH 7.4, pelleted, and stored at $-70^{\circ} \mathrm{C}(3)$. This partial purification results in $P$. carinii preparations of $>90-95 \%$ purity, with the remaining cells being host cells. An aliquot was cultured for bacteria and fungi, and preparations with bacterial ( $>10$ colonies) or fungal contamination were not used. Human $P$. carinii were obtained from autopsy lung specimens from patients with AIDS and $P$. carinii pneumonia, and were stored frozen before processing. Human $P$. carinii were processed in a manner identical to rat $P$. carinii, except that density gradient centrifugation was omitted (3). Density gradient centrifugation was omitted in processing human $P$. carinii because this step does not increase purity but decreases yield when using previously frozen organisms.

Antigen solubilization. Pellets of organisms were resuspended in 5-10 ml of $1 \mathrm{M}$ sorbitol, $1 \mathrm{mM}$ EDTA, pH 8.5, and zymolyase (ICN Biomedicals, Inc., Costa Mesa, CA), $1 \mathrm{mg} / \mathrm{ml}$, or PBS containing 100 $\mathrm{U} / \mathrm{ml}$ lyticase (Sigma Chemical Co., St. Louis, MO) $(9,13)$. The suspensions were incubated for $18 \mathrm{~h}$ at $37^{\circ} \mathrm{C}$ and the supernatant was clarified by centrifugation $(2,000 \mathrm{~g})$ for $10 \mathrm{~min}$. Preparations were concentrated using a microconcentrator (Centricon-30; Amicon Corp., Danvers, MA).

Polyacrylamide gel electrophoresis and immunoblotting. SDSPAGE was performed as previously described $(2,3,14)$ based on the method of Laemmli, using a $4 \%$ stacking gel and an 8 or $10 \%$ running gel. The stacking gel buffer was $0.125 \mathrm{M}$ Tris- $\mathrm{HCl}, \mathrm{pH} 6.8$, the running gel buffer was $0.475 \mathrm{M}$ Tris- $\mathrm{HCl}, \mathrm{pH} 8.8$, and the electrode buffer was Tris-glycine, pH 8.3 (0.25 M Tris, $0.192 \mathrm{M}$ glycine, and $0.1 \% \mathrm{SDS})$. Samples for SDS-PAGE were prepared by heating $\left(100^{\circ} \mathrm{C}\right.$ for $\left.5 \mathrm{~min}\right)$ in $0.125 \mathrm{M}$ Tris- $\mathrm{HCl}(\mathrm{pH} \mathrm{6.8)}$ in the presence of $2 \% \mathrm{SDS}$ and 5\% 2-mer-

1. Abbreviations used in this paper: BS3, bis-(sulfosuccinimidyl) substrate; gp, glycoprotein; TBS, Tris-buffered saline. 
captoethanol. Gels were run at $10^{\circ} \mathrm{C}$ with $60 \mathrm{~mA}$ constant current, using bromphenol blue as a marker.

Following separation by SDS-PAGE, the gels were either stained with Coomassie blue or a silver stain (Bio-Rad Laboratories, Richmond, CA) or proteins were electrophoretically transferred to nitrocellulose and evaluated by the Western blot (immunoblot) technique (2, 3,15 ). For transfer, gels were preequilibrated for $30 \mathrm{~min}$ in Tris-glycine (pH 8.3) in $20 \%$ methanol and transferred overnight in the same buffer at $4^{\circ} \mathrm{C}$ by using an electrophoretic blotting apparatus (Bio-Rad) set at $30 \mathrm{~V}$. After transfer, the nitrocellulose was blocked by incubation for $60 \mathrm{~min}$ in $3 \%$ gelatin in Tris-buffered saline (TBS; $0.02 \mathrm{M}$ Tris, $0.5 \mathrm{M}$ $\mathrm{NaCl} \mathrm{pH} 7.5$ ), and washed in TBS containing $0.05 \%$ Tween 20 . The nitrocellulose was then incubated for $2 \mathrm{~h}$ at room temperature with the first antibody. Antibody preparations used at this step included human serum (1:100 dilution) from patients with and without $P$. carinii pneumonia, serum from a rat immunized with rat $P$. carinii, ascites from a mouse that had been immunized with human $P$. carinii and had subsequently spontaneously developed ascites, and ascites containing MAb 6B8 (3). The first ascites reacted with multiple human $P$. carinii antigens, including the $95,000-\mathrm{mol} w \mathrm{t}$ antigen. MAb 6B8 reacts exclusively with the latter antigen (3). Following incubation, the blots were washed, incubated ( $2 \mathrm{~h}$, room temperature) with horseradish peroxidase-conjugated goat antibody to rat IgG, mouse IgG, or human IgG (Bio-Rad), washed, and incubated with 4-chloro-1-naphthol (Bio-Rad) in the presence of $0.015 \% \mathrm{H}_{2} \mathrm{O}_{2}$.

High performance liquid chromatography. Antigens were purified by HPLC using a molecular sieve column followed by an ion exchange column attached to an HPLC apparatus (Waters Chromatography Division, Millipore Corp., Milford, MA). Antigens were first separated by size using a Spherogel-TSK G2000SW column (Beckman Instruments, Inc., Fullerton, CA) under isocratic conditions at a flow rate of 0.5 $\mathrm{ml} / \mathrm{min}$ using $0.1 \mathrm{M} \mathrm{KPO4}, 0.2 \mathrm{M} \mathrm{NaCl}, \mathrm{pH} 7.0$, as the running buffer. Fractions were collected every minute, and aliquots were evaluated by SDS-PAGE and immunoblot. Fractions containing the antigen were pooled, washed with $0.01 \mathrm{M}$ Tris $\mathrm{pH} 7.4$, and concentrated using a Centriprep 10 concentrator (Amicon). The preparation was then applied to a Spherogel-TSK DEAE-3SW column (Beckman) and resolved by gradient chromatography using a linear gradient from 0 to $0.5 \mathrm{M} \mathrm{NaCl}$ in $0.01 \mathrm{M}$ Tris, $\mathrm{pH} 7.4$, at a flow rate of $1 \mathrm{ml} / \mathrm{min}$. Fractions were collected every minute and aliquots were evaluated by SDSPAGE and immunoblotting. The antigens were concentrated as above and washed with $40 \times$ vol of PBS.

Deglycosylation. Antigen preparations were dissolved in $0.05 \mathrm{M}$ sodium acetate, $\mathrm{pH} 5.5,0.1 \% \mathrm{SDS}$, and heated $\left(100^{\circ} \mathrm{C}\right)$ for $3 \mathrm{~min} ; 20 \mu \mathrm{l}$ (0.02 U) endoglycosidase $\mathbf{H}$ (Boehringer Mannheim Biochemicals, Indianapolis, IN) was added and the sample was incubated for $24 \mathrm{~h}$ at $37^{\circ} \mathrm{C}$; this process was repeated $(16,17)$.

Glycoprotein detection. Following transfer of SDS-PAGE-separated antigens to nitrocellulose, blots were blocked for $1 \mathrm{~h}$ at room temperature with 3\% BSA in PBS. Blots were incubated for $1 \mathrm{~h}$ at room temperature in PBS and Con A $(10 \mu \mathrm{g} / \mathrm{ml})$ (Sigma) with and without $100 \mathrm{mM} \alpha$-methylmannoside (Sigma) and washed twice (5 min each) with PBS. Blots were then incubated for $1 \mathrm{~h}$ with horseradish peroxidase $(5 \mathrm{mg} / \mathrm{ml})$ (Cooper Biomedical, Inc., Malvern, PA) in PBS, washed twice (5 min each) with PBS, and developed with 4-chloro-1naphthol in the presence of $0.015 \% \mathrm{H}_{2} \mathrm{O}_{2}(18)$.

Carbohydrate analysis. Purified $P$. carinii protein samples (15-50 $\mu \mathrm{g})$ were taken to dryness, dissolved in $100 \mu \mathrm{l}$ of $4.2 \mathrm{M}$ trifluoroacetic acid, and heated at $100^{\circ} \mathrm{C}$ for $4 \mathrm{~h}$. The acid was removed under a stream of nitrogen, and the residue taken up in an equal amount of water that was again blown to dryness. The final residue was dissolved in 100-500 $\mu \mathrm{l}$ of water and aliquots were analyzed by HPLC. The analysis was carried out by high-pH anion exchange chromatography on a BioLc Carbohydrate Analyzer (Dionex Corp., Sunnyvale, CA) employing a CarboPacPA-1 column $(4.6 \times 250 \mathrm{~mm})$ and $10 \mathrm{mM}$ $\mathrm{NaOH}$ as eluent. The several monosaccharides were monitored by a sensitive pulsed amperometric detector and recovered as well-separated individual peaks.
Cross-linking with bis-(sulfosuccinimidyl) substrate (BS3). Crosslinking experiments were performed using solubilized crude or partially purified antigens $(20 \mu \mathrm{g})$ in PBS, using BS3 (0.1-1.0 mM) (Pierce Chemical Co., Rockford, IL) as the cross-linking agent (19). Preparations were incubated at room temperature for $18 \mathrm{~h}$ and the reaction was stopped by the addition of 0.1 vol $50 \mathrm{mM}$ 2-amino-ethanol (Sigma). Results were evaluated by SDS-PAGE and immunoblotting.

IgG ELISA. 48 wells of a 96-well microtiter plate (MaxiSorp Immuno Plate; Nunc, Roskilde, Denmark) were coated overnight at $4^{\circ} \mathrm{C}$ with $100 \mu \mathrm{l}$ purified human $P$. carinii antigen $(0.5 \mu \mathrm{g} / \mathrm{ml})$ in $0.5 \mathrm{M}$ carbonate buffer, $\mathrm{pH}$ 9.6. The remaining wells were coated with carbonate buffer alone. The plates were washed six times with PBS containing $0.05 \%$ Tween-20, blocked for $1 \mathrm{~h}$ at $37^{\circ} \mathrm{C}$ with $200 \mu \mathrm{l} 5 \%$ goat serum (Gibco Laboratories, Grand Island, NY) in PBS, and washed as before. 100- $\mu$ l aliquots of human serum samples diluted (1:100) in PBS plus $5 \%$ goat serum and $0.01 \%$ Tween-20 were added to each well and plates were incubated for $1 \mathrm{~h}$ at $37^{\circ} \mathrm{C}$. All samples were run in triplicate, and were also run in triplicate against the wells containing carbonate buffer alone. Controls on each run included a known positive sample and a known negative sample. Following washing, $100 \mu \mathrm{l}$ of peroxidase-conjugated goat anti-human IgG (heavy and light chain specific) (Jackson Immuno Research Laboratories, Inc., West Grove, PA) diluted 1:50,000 in PBS-goat serum-Tween 20 was added to each well. After incubation at $37^{\circ} \mathrm{C}$ for $1 \mathrm{~h}$, the plates were washed and $100 \mu \mathrm{l}$ of $0.1 \% o$-phenylenediamine (Sigma) in the presence of $0.003 \% \mathrm{H}_{2} \mathrm{O}_{2}$ was added to each well. After $40 \mathrm{~min}$ at room temperature, the reaction was stopped by addition of $50 \mu 120 \% \mathrm{H}_{2} \mathrm{SO}_{4}$, and the absorbance was determined on a dual wavelength reader (MR 700; Dynatech Laboratories, Inc., Dynatech Corp., Chantilly, VA), using $630 \mathrm{~nm}$ as reference and $490 \mathrm{~nm}$ as the test wavelength. Optical density values for each sample were determined by subtracting the mean optical density of the triplicate wells containing no antigen from the mean of the triplicate wells containing purified protein. Optical density results were interpreted as follows: $<0.150$, negative; $0.150-0.199$, indeterminate; $\geq 0.200$, positive.

Protein determination. Protein concentration was determined by a colorimetric assay according to the manufacturer's instructions, using BSA as the standard (BCA protein assay reagent; Pierce).

\section{Results}

Previous observations that the major rat $P$. carinii antigen, gp 116, can be solubilized by treatment with zymolyase were confirmed in this study (Fig. 1) (9). The human $P$. carinii protein of $95,000 \mathrm{~mol} w \mathrm{t}$ was also readily solubilized by treatment with zymolyase or lyticase, which is a similar enzyme (Fig. 1). To document that this observation was not limited to a single human $P$. carinii isolate, five additional human $P$. carinii isolates were treated in an identical manner; for each isolate a similar antigen was solubilized (gel not shown). Solubilization resulted in a small decrease in molecular weight of the rat $P$. carinii, but not human $P$. carinii, protein (Fig. 1). Following solubilization, the antigen retained its reactivity with $\mathrm{MAb}$ $6 \mathrm{~B} 8$, and this antibody together with polyclonal mouse antibodies was used to identify the antigen during purification, for which a single human $P$. carinii isolate was used. Because no monoclonal antibody to the rat $P$. carinii antigen was available, polyclonal rat serum was used to identify the rat antigen.

Purification was performed in two steps. After solubilization, the antigens were partially purified by HPLC using a molecular sieve column. Both the rat and the human $P$. carinii antigen were eluted, as determined by SDS-PAGE and immunoblotting, in the void volume, and each had an apparent mol wt of $>290,000$. Following ion exchange chromatography, the proteins were pure as determined by SDS-PAGE, and re- 

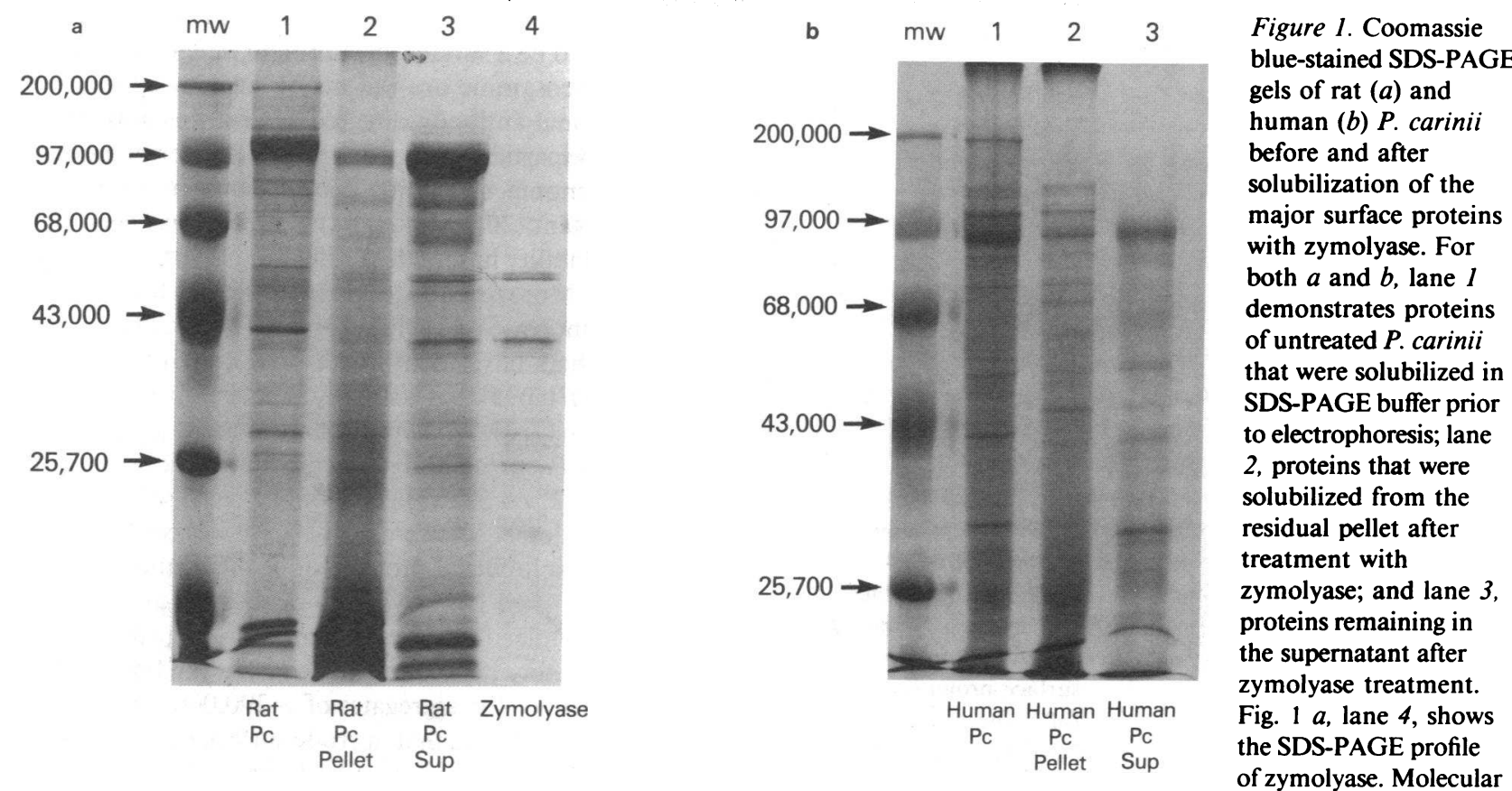

weight markers indicated on the left include myosin $(200,000)$, phosphorylase B $(97,000)$, BSA $(68,000)$, ovalbumin $(43,000)$, and $\alpha$-chymotrypsinogen $(25,700)$.

mained reactive with antibody $6 \mathrm{~B} 8$ or the polyclonal antibodies (Fig. 2).

During purification by the sizing column the native form of the human $P$. carinii surface antigen had an apparent $\mathrm{mol}$ wt of $>290,000$, rather than 95,000 as was found under denaturing and reducing conditions on the SDS-PAGE gels. To determine whether the native antigen existed as a multimer, an attempt was made to cross-link the antigen with BS3 before denaturation and reduction. Cross-linking resulted in a decrease in the intensity of the $95,000-\mathrm{mol}$ wt band, and the formation of a broad protein band of $\sim 250,000-300,000 \mathrm{~mol}$ wt (Fig. 3). When rat gp 1 16, which in native form elutes in a manner similar to the human $P$. carinii antigen, was cross-linked, a similar band was seen (Fig. 3). Immunoblots demonstrated reactivity of the new bands with polyclonal antibodies (Fig. 3 ) as well as MAb 6B8 (immunoblot not shown).

Previous investigations have demonstrated that rat $P$. carinii gp1 16 is a surface glycoprotein and that at least a portion of the carbohydrate moiety is susceptible to treatment with endoglycosidase $\mathbf{H}(7-11)$. The human $P$. carinii antigen was also found to be a glycoprotein susceptible to endoglycosidase $\mathrm{H}$ treatment. Treatment with this enzyme lead to a reduction in mol wt of $\sim 9 \%$ for both rat and human antigens (Fig. 4). Con A bound to this antigen, and treatment with endoglycosidase $H$ abolished this binding (Fig. 4). Despite the loss of reactivity with Con A, immunoreactivity with either polyclonal or monoclonal antibodies was retained following deglycosylation (blots not shown).

To further investigate the sugar composition of the surface antigens, carbohydrate analysis using high $\mathrm{pH}$ anion chromatography was performed. Two different purified human $P$. $c a$ rinii antigens and a purified rat $P$. carinii antigen were analyzed by this technique. For both human $P$. carinii antigens, the major sugars were mannose, glucose, galactose, and glucosamine, present in approximately equimolar ratios (Fig. 5). For the rat $P$. carinii antigen, mannose and glucose were also present in approximately equimolar ratios, but glucosamine and galactose were each present at one-fifth the concentration of mannose or glucose (Fig. 5). Because sialic acid is destroyed by trifluoroacetic acid, the sialic acid content of the glycoproteins could not be determined by this procedure.

To evaluate the immune response of humans to gp95, an ELISA was developed, as described in the methods, for detection of IgG antibodies to the purified antigen. Optimal conditions were determined by preliminary experiments. To validate the reliability of the ELISA, 31 samples were evaluated in parallel ELISA and immunoblot assays (Fig. 6). A sample was considered positive by immunoblot if any band of $95,000 \mathrm{~mol} \mathrm{wt}$ was seen. In 25 samples, there was agreement between ELISA and immunoblot results; among the remaining 6 samples, all of which were indeterminate by ELISA, 2 were positive and 4 were negative by immunoblot. The patient from whom the human $P$. carinii isolate used in these studies was obtained demonstrated reactivity with gp 95 by both ELISA and immunoblot. Additional sera from healthy and immunosuppressed humans were then evaluated by ELISA, and results are summarized in Table I. Among 27 infants hospitalized for diarrhea, one value was indeterminate, and the remaining 26 were negative. No healthy adult laboratory worker had detectable antibodies against the gp95 antigen. Among AIDS and non-AIDS immunocompromised patients with no history of $P$. carinii pneumonia, only 1 of 16 sera were positive by ELISA. Among 30 AIDS and non-AIDS patients with a history of $P$. carinii pneumonia, 10 patients had at least 1 sample positive for antibodies to gp 95 by ELISA, 7 patients had indeterminate samples, and 13 had negative samples. When sera obtained only within $3 \mathrm{wk}$ of an episode of $P$. carinii pneumonia were evaluated, 7 samples were positive, 5 were indeterminate, and 12 

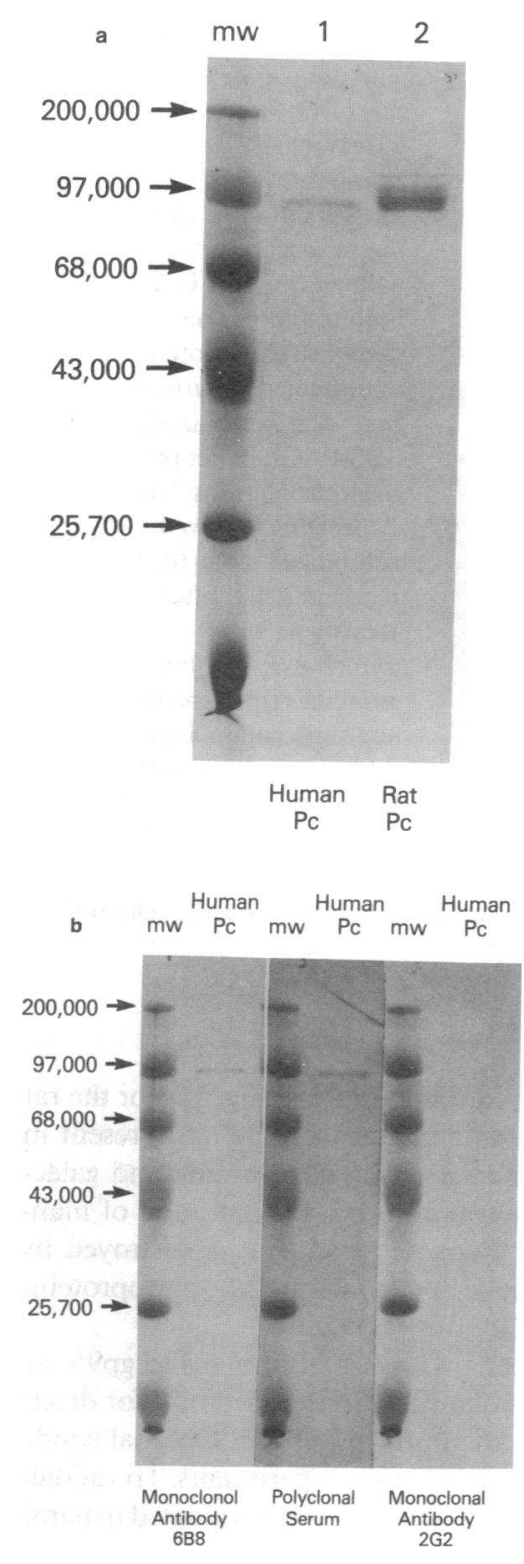

Figure 2. (a) Coomassie blue-stained SDSPAGE gel of purified human (lane 1 ) and rat (lane 2) P. carinii surface proteins following molecular sieve and ion-exchange chromatography. (b) Immunoblot analysis of human $P$. carinii gp95 after purification. Panels 1 and 2 show reactivity of the purified antigen with MAb 6B8 and polyclonal antibodies, respectively. For panel 3, which shows no reactivity, the blot was incubated with MAb 2G2, which is specific for a 65,000 mol wt antigen of human $P$. carinii. Molecular weight markers indicated on the left are the same as for Fig. 1.

were negative. Among six AIDS patients with a history of $P$. carinii pneumonia for whom serial serum samples were available before and after the episode of pneumonia, no consistent pattern of reactivity was seen. Some patients developed antibodies to gp 95 before or concomitant with the development of clinical disease, others lost antibodies before development of overt pneumonia, and still others never developed detectable antibodies despite multiple episodes (Fig. 7).

\section{Discussion}

Studies of the epidemiology and immunology of $P$. carinii infection in humans have been hampered by an inability to obtain purified human $P$. carinii antigens in sufficient quantities. Although the immunoblot technique has recently been successfully used by our group as well as other investigators to address these issues (2-6), purified antigens would greatly facilitate such studies. By SDS-PAGE and immunoblot, a major rat $P$. carinii antigen of 110,000-120,000 mol wt (gp 1 16) has been identified by numerous investigators $(2,7-11)$. This antigen has been found to be a surface glycoprotein in which mannose and $N$-acetylglucosamine are the major sugar residues $(7,9$, 11). A monoclonal antibody directed against this antigen has been found to be partially protective in a rat and ferret model of $P$. carinii pneumonia, suggesting that this antigen is immunologically important (20). Although this protein has been purified, lack of solubility has made purification of large quantities difficult $(7,11)$. More recently, other investigators have demonstrated that zymolyase can solubilize this antigen (9).

We and others have identified a predominant protein of human $P$. carinii (gp95) that is slightly smaller than the predominant rat antigen $(2,3)$. We hypothesized that the predominant rat and human $P$. carinii antigens were homologous, and thus that the human $P$. carinii antigen would also be solubilized by zymolyase or lyticase. After preliminary studies confirmed this hypothesis, the solubilized proteins of both rat and human $P$. carinii were purified by a two-step procedure and partially characterized. Molecular sieve and cross-linking studies demonstrated that before reduction and denaturation both proteins existed as multimers or aggregates of $>290,000$ mol wt. Radding et al. similarly found that in crude surface protein preparations the major surface protein of rat $P$. carinii had an apparent mol wt of $>1,000,000$ (7). The lower molecular weight we observed following cross-linking (250,000-300,000 mol wt) may be due to inefficient cross-linking or to different solubilization methods.

Lectin studies have previously shown that the predominant antigens of both rat and human $P$. carinii are glycoproteins that contain mannose and $\mathrm{N}$-acetylglucosamine as the major carbohydrates $(9,10,21)$. This study confirms that these antigens are glycoproteins that are susceptible to endoglycosidase $\mathrm{H}$; following treatment with this enzyme, there was an $\sim 9 \%$ decrease in the molecular weight of both glycoproteins, similar to results previously reported for the rat antigen. Identical results were seen with endoglycosidase F (results not shown). Radding et al. previously found, however, that such deglycosylation was incomplete, and following chemical deglycosylation of gp116 with trifluoromethanesulfonic acid, the major core protein had a mol wt of 68,000 (7). Attempts to deglycosylate the human $P$. carinii gp95 with trifluoromethanesulfonic acid were unsuccessful in our study. Susceptibility to endoglycosidase $\mathrm{H}$ and $\mathrm{F}$ demonstrate that both rat and human $P$. carinii glycoproteins contain high-mannose or possibly mixed oligosaccharides that are $\mathrm{N}$-linked to the core protein. Since reactivity with Con A was eliminated by treatment of the proteins with endoglycosidase $\mathrm{H}$ (Fig. 4), any carbohydrate moiety remaining after such treatment is either not reactive with or inaccessible to Con A.

Carbohydrate analysis of rat and human $P$. carinii glycoproteins demonstrated distinct differences. Whereas in two human $P$. carinii isolates mannose, glucose, galactose, and glucosamine were present in equimolar concentrations, in the rat $P$. carinii glycoprotein glucose and mannose were present in fivefold higher concentrations than the two other carbohydrates. Tanabe et al., using less sensitive techniques, previously found mannose to be the predominant carbohydrate of the major rat $P$. carinii glycoprotein (11). The occurrence in our study of sugars other than mannose in high molar ratios suggests that in addition to the high-mannose residues, mixed or complex $\mathrm{N}$ linked or O-linked oligosaccharides also are present. The presence of glucose in the glycoproteins is unusual, and raises the question of an artifactual result. However, no glucose-contain- 


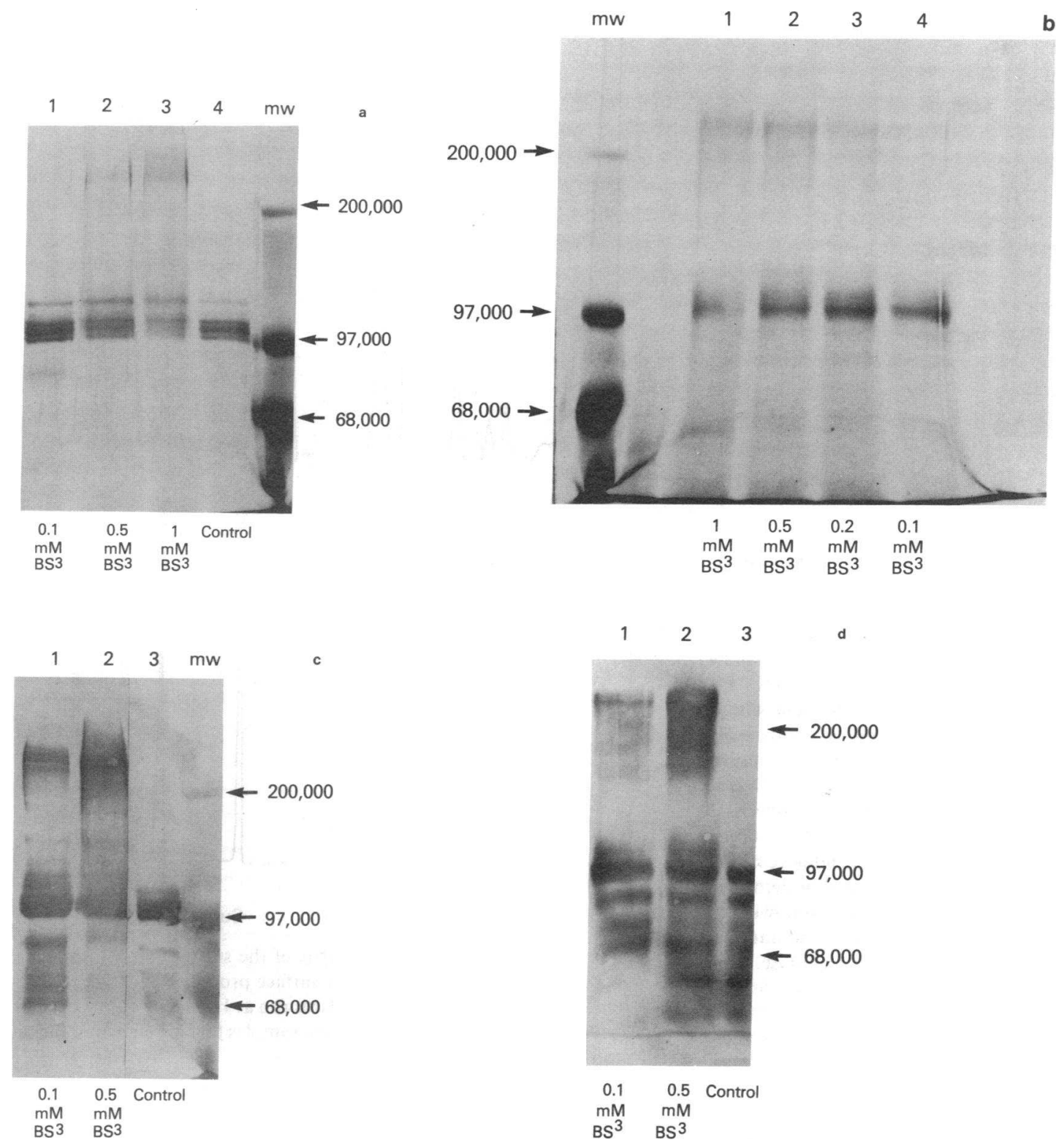

Figure 3. (a) and (b) Demonstration of the appearance of a new, high molecular weight band following cross-linking of rat $(a)$ and human $(b) P$. carinii proteins by BS3. Partially purified antigen preparations were cross-linked with BS3; proteins were then separated by SDS-PAGE, and the gel was stained with Coomassie blue. The concentration of BS3 is indicated at the bottom of the figures. As the concentration of BS3 was increased, the intensity of the original band decreased, and the intensity of the new band of 250,000-300,000 increased. $(c)$ and $(d)$ Immunoblots demonstrating the immunoreactivity of the new, high molecular weight bands of rat $(c)$ and human $(d) P$. carinii. The concentrations of BS3 are indicated at the bottom.

ing reagent was used during the purification, suggesting that the observation is real.

Susceptibility of $P$. carinii to zymolyase and the presence of glycoproteins containing high-mannose residues suggests a structural similarity to Saccharomyces cerevisiae (22). Zymolyase contains two enzymatic activities, a $\beta-1,3$ glucanase as well as a protease, both of which are necessary for lysis of yeast (13); this study cannot determine which of these activities is important in solubilizing the surface proteins. Previous investigators have suggested that $\beta-1,3$ glucan is present in the cell wall of $P$. carinii, and recent studies indicate that $P$. carinii may contain $\beta$-glucan synthase activity, further suggesting a similarity to yeast $(9,23)$. It is possible that the glucose detected in the purified glycoprotein preparations is derived from the glucan matrix.

To evaluate the immune response to human $P$. carinii gp95, an ELISA using purified protein was developed. Previous studies using immunofluorescence or the immunoblot technique have shown that most adults have antibodies to human $P$. carinii $(2,24-28)$. We and others have found that despite the abundance of gp95 (as evaluated by Coomassie blue stain), it does not appear by immunoblot to be an immunodominant protein $(2,4,29)$. Rather, most humans have antibodies to a broad band of 35,000-45,000 mol wt that cannot be identified on Coomassie blue-stained gels. This study supports these observations. Only one individual without a history of $P$. carinii 


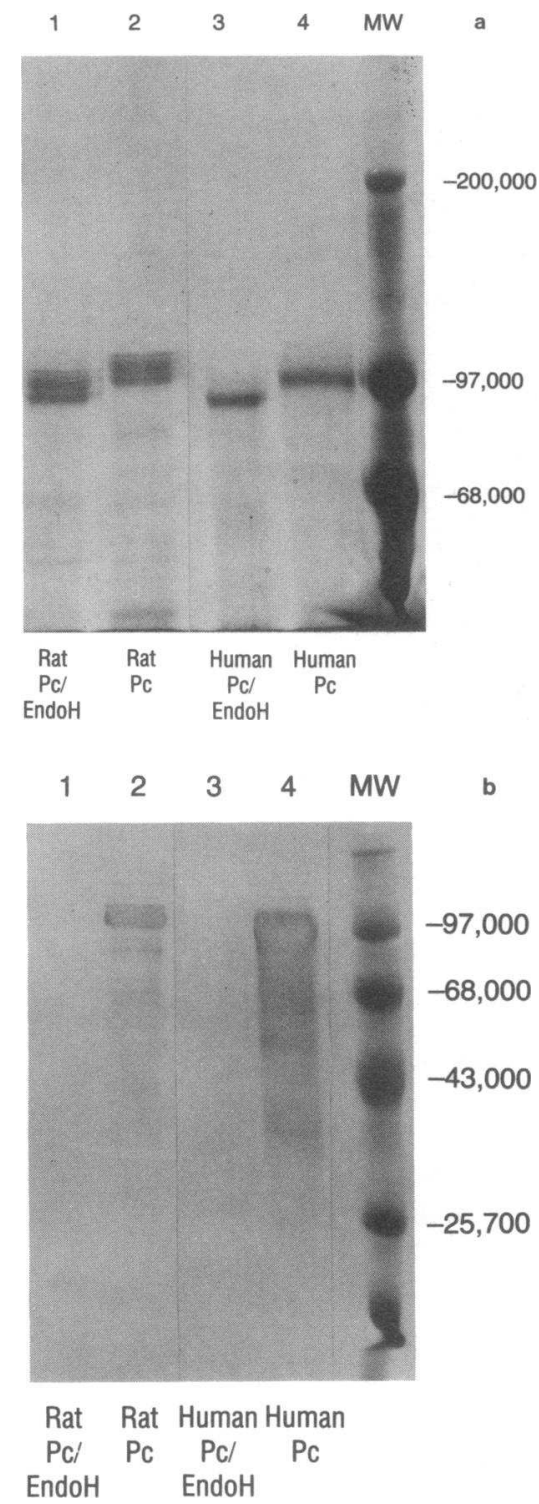

Rat P. carinii gp116

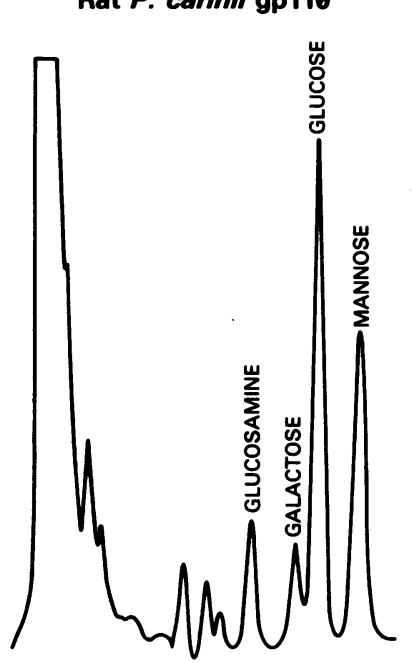

Figure 4. (a) Silver stained SDS-PAGE gel of rat and human $P$. carinii surface antigens before (lanes 2 and 4) and after (lanes $l$ and 3) treatment with endoglycosidase $\mathrm{H}$. A reduction in molecular weight of $\sim 9 \%$ is seen for both proteins. (b) Reactivity of untreated and endoglycosidasetreated proteins with Con A following SDSPAGE and transfer to nitrocellulose. There is loss of reactivity with Con A for both proteins following enzyme treatment.

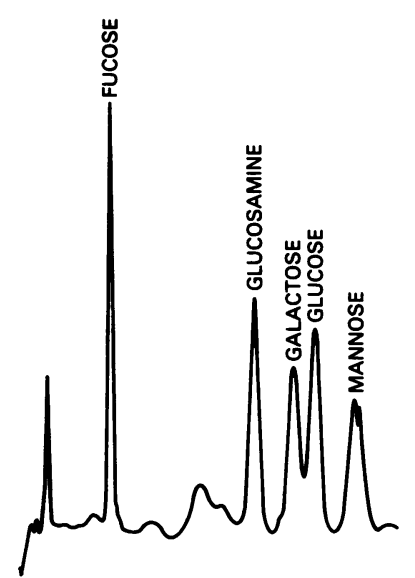

Sugar Standards top chromatograms.
Human P. carinii gp95
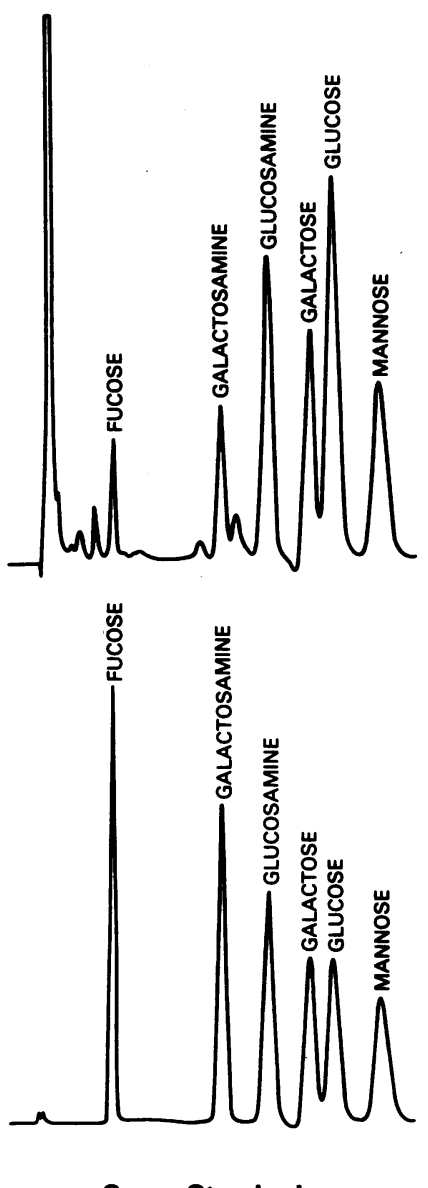

Sugar Standards

Figure 5. Chromatographic analysis of the sugar composition of purified rat and human $P$. carinii surface proteins. The two bottom chromatograms are of sugar standards run at $1-\mathrm{nmol}$ levels in parallel with the rat and the human $P$. carinii samples that are shown in the

Table I. gp95 ELISA Results

\begin{tabular}{|c|c|c|c|c|c|}
\hline Patient population & History of $P$. carinii pneumonia & $\begin{array}{l}\text { Positive } \\
n(\%)\end{array}$ & $\begin{array}{c}\text { Indeterminate } \\
n(\%)\end{array}$ & $\begin{array}{c}\text { Negative } \\
n(\%)\end{array}$ & Total \\
\hline Infants & No & $0(0)$ & $1(4)$ & $26(96)$ & 27 \\
\hline Healthy laboratory workers & No & $0(0)$ & $0(0)$ & $8(100)$ & 8 \\
\hline \multirow{3}{*}{ Non-AIDS, immunosuppressed } & No & $0(0)$ & $1(33)$ & $2(67)$ & 3 \\
\hline & Yes & $4(33)$ & $3(25)$ & $5(42)$ & 12 \\
\hline & Yes, within $3 \mathrm{wk}$ & $4(40)$ & $2(20)$ & $4(40)$ & 10 \\
\hline \multirow[t]{3}{*}{ AIDS } & No & $1(20)$ & $1(20)$ & $3(60)$ & 5 \\
\hline & Yes & $6(33)$ & $4(22)$ & $8(45)$ & 18 \\
\hline & Yes, within 3 wk & $3(21)$ & $3(21)$ & $8(58)$ & 14 \\
\hline
\end{tabular}

Detection of IgG to gp95 by ELISA for the indicated patient populations. Serum (diluted 1:100) was evaluated in triplicate, and the mean of the results was used to categorize reactivity. Patients were categorized as having a history of $P$. carinii pneumonia if they had any history of this infection. Patients with $P$. carinii pneumonia within $3 \mathrm{wk}$ of obtaining the evaluated serum sample are a subpopulation of all patients with $P$. carinii pneumonia. 


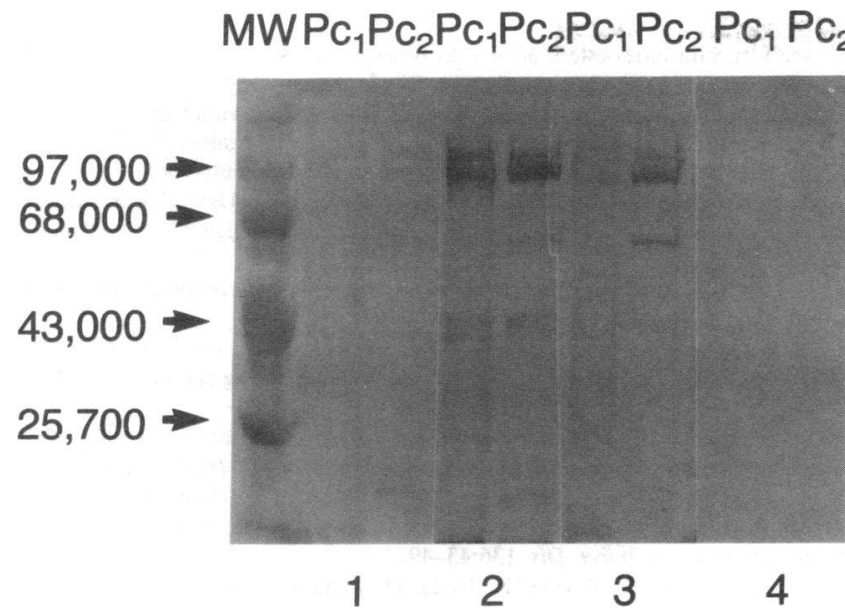

Figure 6. Immunoblots of two partly purified human $P$. carinii samples that were reacted with four sera (1:100 dilution) obtained from the following patients: 1 , non-AIDS immunosuppressed patient with $P$. carinii pneumonia; 2 and 3, AIDS patients with $P$. carinii pneumonia; 4 , healthy laboratory worker. The first three serum samples, which react with gp 95 by immunoblot, were positive in the gp95 ELISA, and the fourth sample was negative by both immunoblot and ELISA.

pneumonia (a patient with HIV infection and a history of Kaposi's sarcoma) of 43 tested had clearly detectable antibodies to gp95, and three had indeterminate results. 10 of 30 immunocompromised patients with a history of $P$. carinii pneumonia had detectable antibodies, and an additional 7 had indeterminate results. In patients with $P$. carinii pneumonia for whom serial samples were available, variable responses were seen. Some patients developed no antibody response despite multiple episodes of pneumonia, others developed antibodies after clinical disease, and yet others developed detectable antibodies before diagnosis of $P$. carinii pneumonia (Fig. 7). Larger studies evaluating the usefulness of antibody detection are needed, but the current study suggests that in a subpopulation of patients clinical $P$. carinii pneumonia may be heralded by the development of such antibodies. Whether such antibodies are predictive of a favorable outcome cannot be evaluated by this study, but as previously noted, monoclonal antibodies to the analogous ferret and rat antigen may be protective (20).

It is unclear why so few humans have antibodies to gp95. It is possible that antigenic variability, with little cross-reactivity, occurs among different human $P$. carinii strains. However, at least one epitope, that identified by antibody $6 \mathrm{~B} 8$, reacts with all isolates tested to date, and human sera that react by immunoblot with one isolate also react with other isolates (3). Alternatively, the antigen may not be exposed to the host immune system, although immunofluorescent studies demonstrate surface reactivity with $6 \mathrm{~B} 8$. It is also possible that the antigen is structurally related to host antigens resulting in tolerance.

The availability of purified $P$. carinii surface antigens will allow more precise investigations of the function of these proteins and the host immune response to them. In preliminary studies, no blastogenic response to gp 95 by peripheral blood mononuclear cells obtained from normal hosts was seen. Previous investigators have demonstrated blastogenic responses to $P$. carinii in healthy adults, using crude antigen preparations $(30-32)$. Since the antigens used in those studies were

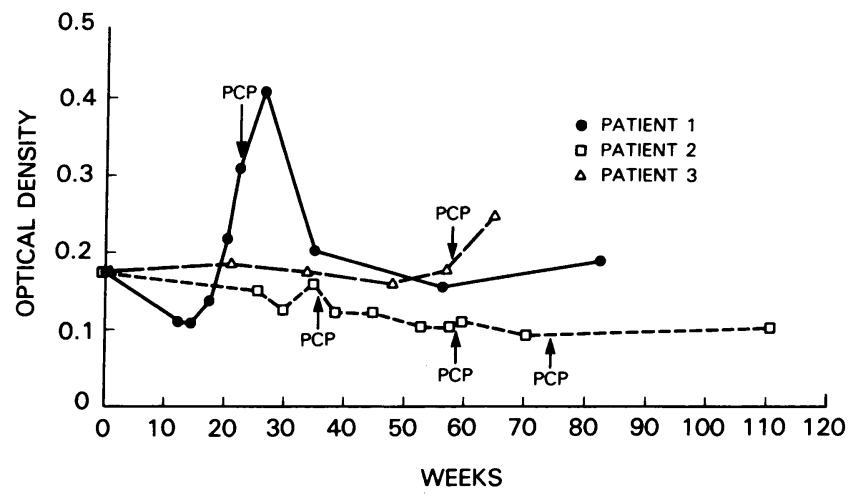

Figure 7. Changes in ELISA values with time in three patients with $P$. carinii pneumonia. Serial samples were available for these patients before and after episodes of $P$. carinii pneumonia, which are indicated by the arrows. Weeks after obtaining the first serum sample are shown along the bottom, and optical density is shown along the left. Patient 1 had an increase in optical density prior to the development of $P$. carinii pneumonia. Patient 2 had no change in optical density despite three episodes of $P$. carinii pneumonia, and patient 3 had an increase after developing pneumonia.

not purified or well characterized, it is possible that the blastogenic responses were nonspecific. Alternatively, the blastogenic responses may not have been to the major surface antigen, but to other, more immunogenic, antigens. Further studies to evaluate blastogenic responses in patients at risk for $P$. carinii pneumonia may help explain the role of $T$ cell-mediated immunity in protecting against this infection.

The role of the major surface glycoprotein of $P$. carinii is unknown; speculation has centered on mediating attachment to host cells or on a role in evasion of host defense mechanisms (7, 11). Recent studies, for example, have suggested that $P$. carinii attachment to tissue culture cells is mediated by fibronectin, and that rat $P$. carinii gp1 16 is the receptor for this attachment $(33,34)$. Whether the human $P$. carinii antigen interacts with fibronectin or with other cell receptors, such as the recently described mannose-binding protein, remains to be determined by further investigations $(35,36)$. Nonetheless, the distinctive carbohydrate profiles of $P$. carinii isolated from different species and the previously documented antigenic differences between rat and human isolates suggest that this antigen plays a role in determining host specificity or host-organism interactions.

In summary, we have purified and partially characterized major surface antigens of rat and human $P$. carinii. Extension of these studies will help us to better understand this pathogen, and ultimately may lead to improved management of $P$. carinii pneumonia.

\section{Acknowledgments}

We would like to thank Dr. Gilbert Ashwell for performing the carbohydrate analyses that were essential to this study, and Dr. Andrea Kovacs for supplying the infant sera.

Bettina Lundgren was supported in part by the Danish Medical Research Council.

\section{References}

1. Kovacs, J. A., J. W. Hiemenz, A. M. Macher, D. Stover, H. W. Murray, J. Shelhamer, H. C. Lane, U. Urmacher, C. Honig, D. L. Longo, et al. 1984. Pneumocystis carinii pneumonia: a comparison between patients with the acquired 
immunodeficiency syndrome and patients with other immunodeficiencies. Ann. Intern. Med. 100:663-671.

2. Kovacs, J. A., J. L. Halpern, J. C. Swan, J. Moss, J. E. Parrillo, and H. Masur. 1988. Identification of antigens and antibodies specific for Pneumocystis carinii. J. Immunol. 140:2023-2031.

3. Kovacs, J. A., J. L. Halpern, B. Lundgren, J. C. Swan, J. E. Parrillo, and H. Masur. 1989. Monoclonal antibodies to Pneumocystis carinii: identification of specific antigens and characterization of antigenic differences between rat and human isolates. J. Infect. Dis. 159:60-70.

4. Walzer, P. D., and M. J. Linke. 1987. A comparison of the antigenic characteristics of rat and human Pneumocystis carinii by immunoblotting. J. Immunol. 138:2257-2265.

5. Graves, D. C., S. J. McNabb, M. H. Ivey, and M. A. Worley. 1986. Development and characterization of monoclonal antibodies to Pneumocystis carinii. Infect. Immun. 51:125-133.

6. Graves, D. C., S. J. McNabb, M. A. Worley, T. D. Downs, and M. H. Ivey. 1986. Analyses of rat Pneumocystis carinii antigens recognized by human and rat antibodies by using western immunoblotting. Infect. Immun. 54:96-103.

7. Radding, J. A., M. Y. K. Armstrong, E. Ullu, and F. F. Richards. 1989. Identification and isolation of a major cell surface glycoprotein of Pneumocystis carinii. Infect. Immun. 57:2149-2157.

8. Gigliotti, F., L. R. Ballou, W. T. Hughes, and B. D. Mosley. 1988. Purification and initial characterization of a ferret Pneumocystis carinii surface antigen. J. Infect. Dis. 158:848-854.

9. Linke, M. J., M. T. Cushion, and P. D. Walzer. 1989. Properties of the major antigens of rat and human Pneumocystis carinii. Infect. Immun. 57:15471555.

10. Pesanti, E. L., and J. D. Shanley. 1988. Glycoproteins of Pneumocystis carinii: characterization by electrophoresis and microscopy. J. Infect. Dis. 158:1353-1359.

11. Tanabe, K., S. Takasaki, J.-I. Watanabe, A. Kobata, K. Egawa, and Y. Nakamura. 1989. Glycoproteins composed of major surface immunodeterminants of Pneumocystis carinii. Infect. Immun. 57:1363-1368.

12. Masur, H., and T. C. Jones. 1978. The interaction in vitro of Pneumocystis carinii with macrophages and L-cells. J. Exp. Med. 147:157-170.

13. Kitamura, K., and Y. Yamamoto. 1972. Purification and properties of an enzyme, zymolyase, which lyses viable yeast cells. Arch. Biochem. Biophys. 153:403-406.

14. Laemmli, U. K. 1970. Cleavage of structural proteins during the assembly of the head of the bacteriophage T-4. Nature (Lond.). 227:680-685.

15. Towbin, H., T. Staehelin, and J. Gordon. 1979. Electrophoretic transfer of proteins from polyacrylamide gels to nitrocellulose sheets: procedure and some applications. Proc. Natl. Acad. Sci. USA. 76:4350-4354.

16. Maley, F., R. B. Trimble, A. L. Tarentino, and T. H. Plummer, Jr. 1989. Characterization of glycoproteins and their associated oligosaccharides through the use of endoglycosidases. Anal. Biochem. 180:195-204.

17. Trimble, R. B., and F. Maley. 1984. Optimizing hydrolysis of $\mathrm{N}$-linked high-mannose oligosaccharides by endo-beta-N-acetylglucosaminidase H. Anal. Biochem. 141:515-522.

18. Clegg J. C. 1982 . Glycoprotein detection in nitrocellulose transfers of electrophoretically separated protein mixtures using concanavalin A and peroxidase: application to arenavirus and flavivirus proteins. Anal. Biochem. 127:389394.

19. Staros, J. V. 1982. N-hydroxysulfosuccinimide active esters: bis(N-hy- droxysulfosuccinimide) esters of two dicarboxylic acids are hydrophilic, membrane-impermeant, protein cross-linkers. Biochemistry. 21:3950-3955.

20. Gigliotti, F., and W. T. Hughes. 1988. Passive immunoprophylaxis with specific monoclonal antibody confers partial protection against Pneumocystis carinii pneumonitis in animal models. J. Clin. Invest. 81:1666-1668.

21. Yoshikawa, H., T. Tegoshi, and Y. Yoshida. 1987. Detection of surface carbohydrates on Pneumocystis carinii by fluorescein-conjugated lectins. Parasitol. Res. 74:43-49.

22. Cabib, E., R. Roberts, and B. Bowers. 1982. Synthesis of the yeast cell wall and its regulation. Annu. Rev. Biochem. 51:763-793.

23. Williams, D. J., J. A. Radding, M. Y. K. Armstrong, and F. F. Richards. 1990. Pneumocystis carinii contains a $\beta$-glucan synthesizing activity. Clin. Res. 38:363a. (Abstr).

24. Norman, L., and I. G. Kagan. 1973. Some observations on the serology of Pneumocystis carinii infections in the United States. Infect. Immun. 8:317-321.

25. Meuwissen, J. H., I. Tauber, A. D. Leeuwenberg, P. J. Beckers, and M. Sieben. 1977. Parasitologic and serologic observations of infection with pneumocystis in humans. $J$. Infect. Dis. 136:43-49.

26. Tanabe, K., T. Furuta, K. Ueda, H. Tanaka, and K. Shimada. 1985. Serological observations of Pneumocystis carinii infection in humans. J. Clin. Microbiol 22:1058-1060.

27. Pifer, L. L., W. T. Hughes, S. Stagno, and D. Woods. 1978. Pneumocystis carinii infection: evidence for high prevalence in normal and immunosuppressed children. Pediatrics. 61:35-41.

28. Maddison, S. E., G. V. Hayes, S. B. Slemenda, L. G. Norman, and M. H. Ivey. 1982. Detection of specific antibody by enzyme-linked immunosorbent assay and antigenemia by counterimmunoelectrophoresis in humans infected with Pneumocystis carinii. J. Clin. Microbiol. 15:1036-1043.

29. Peglow, S. L., A. G. Smulian, M. J. Linke, C. L. Pogue, S. Nurre, J. Crisler, J. Phair, J. W. M. Gold, D. Armstrong, and P. D. Walzer. 1990. Serologic responses to Pneumocystis carinii antigens in health and disease. J. Infect. Dis. 161:296-306.

30. Herrod, H. G., W. R. Valenski, D. R. Woods, and L. L. Pifer. 1981. The in vitro response of human lymphocytes to Pneumocystis carinii antigen. J. Immunol. 126:59-61.

31. Hagler, D. N., G. S. Deepe, C. L. Pogue, and P. D. Walzer. 1988. Blastogenic responses to Pneumocystis carinii among patients with human immunodeficiency (HIV) infection. Clin. Exp. Immunol. 74:7-13.

32. Hofmann, B., P. B. Nielsen, N. Odum, J. Gerstoft, P. Platz, L. P. Ryder, A. G. Poulsen, L. Mathiesen, E. Dickmeiss, B. Norrild, et al. 1988. Humoral and cellular responses to Pneumocystis carinii, CMV, and herpes simplex in patients with AIDS and in controls. Scand. J. Infect. Dis. 20:389-394.

33. Pottratz, S. T., and W. J. Martin II. 1990. Role of fibronectin in Pneumocystis carinii attachment to cultured lung cells. J. Clin. Invest. 85:351-356.

34. Pottratz, S. T., J. Paulsrud, and W. J. Martin II. 1990. Pneumocystis carinii membrane glycoprotein $\mathrm{gpl} 20$ is a fibronectin binding protein which mediates pneumocystis attachment. Clin. Res. 38:466a. (Abstr).

35. Stahl, P., P. H. Schlesinger, E. Sigardson, J. S. Rodman, and Y. C. Lee. 1980. Receptor-mediated pinocytosis of mannose glycoconjugates by macrophages: characterization and evidence for receptor recycling Cell 19:207-215.

36. Stahl, P. D., J. S. Rodman, M. J. Miller, and P. H. Schlesinger. 1978. Evidence for receptor-mediated binding of glycoproteins, glycoconjugates, and lysosomal glycosidases by alveolar macrophages. Proc. Natl. Acad. Sci. USA. 75:1399-1403. 